Research Article

\title{
The Efficacy of Transarterial Chemoembolization plus Apatinib or Sorafenib in the Treatment of Advanced Hepatocellular Carcinoma
}

\author{
Lei Chen $\mathbb{D}^{1,2,3}$ Tao Sun, ${ }^{1,2,3}$ Linxia Wu, ${ }^{1,2}$ Weihua Zhang, ${ }^{1,2,3}$ Yanqiao Ren, ${ }^{1,2,3}$ \\ Dongqiao Xiang, ${ }^{1,2,3}$ Bin Liang $\mathbb{D},{ }^{1,2,3}$ and Chuansheng Zheng $\mathbb{D}^{1,2,3}$ \\ ${ }^{1}$ Department of Radiology, Union Hospital, Tongji Medical College, Huazhong University of Science and Technology, \\ Wuhan 430022, China \\ ${ }^{2}$ Hubei Province Key Laboratory of Molecular Imaging, Wuhan 430022, China \\ ${ }^{3}$ Department of Interventional Radiology, Union Hospital, Tongji Medical College, \\ Huazhong University of Science and Technology, Wuhan 430022, China \\ Correspondence should be addressed to Bin Liang; bliang@hust.edu.cn and Chuansheng Zheng; hqzcsxh@sina.com
}

Received 22 September 2021; Revised 26 October 2021; Accepted 27 October 2021; Published 19 November 2021

Academic Editor: Ozkan Kanat

Copyright $\odot 2021$ Lei Chen et al. This is an open access article distributed under the Creative Commons Attribution License, which permits unrestricted use, distribution, and reproduction in any medium, provided the original work is properly cited.

\begin{abstract}
Background. Transarterial chemoembolization (TACE) combined with sorafenib (TACE-S) or apatinib (TACE-A) is used in the treatment of hepatocellular carcinoma (HCC). However, to date, no study has compared the efficacy and safety of both treatments. The objective of this study was to compare the efficacy and safety of patients with advanced HCC who received either TACE-S or TACE-A. Methods. 193 patients with advanced HCC were included in the study between June 2015 and December 2019. Propensity score matching (PSM) analysis was used in the study to reduce selection bias. Results. Before PSM, the median overall survival (mOS) and median progression-free survival (mPFS) of patients treated with TACE-S were not significantly longer than in patients treated with TACE-A $(P=0.703, P=0.514)$. TACE-A did not increase the mortality risk compared with TACE-S in the first 12 months (HR: 1.255 , 95\%CI: $0.796-1.978, P=0.329$ ) or after the 12 -month mark (HR: $0.832,95 \%$ CI: $0.482-1.436$, $p=0.508$ ). Similarly, TACE-A did not increase the tumor recurrence risk relative to TACE-S in the first 12 months (HR: 1.054, 95\%CI: $0.744-1.493, P=0.767$ ) or after the 12 -month mark (HR: $1.730,95 \%$ CI: $0.592-5.049, P=0.316$ ). The subgroups analysis showed that TACE-A did not increase mortality risk or tumor recurrence risk relative to TACE-S. After PSM, similar results were presented. The III and IV stage adverse events in the TACE-A group were similar to those in the TACE-S group before PSM. Conclusions. Patients with advanced hepatocellular carcinoma could get similar survival benefits from treatment with either transarterial chemoembolization plus apatinib or transarterial chemoembolization plus sorafenib.
\end{abstract}

\section{Introduction}

Hepatocellular carcinoma (HCC) is one of the most common cancers in the world [1]. At present, there are 841,000 new cases of HCC worldwide every year, and $46.71 \%$ of the new cases are from China [2]. The agestandardized incidence rate per 100,000 is 26.8 in Eastern Asia [1]. Patients with early HCC (Barcelona clinic liver cancer (BCLC) stage 0 or A) have better survival benefits from radical treatment, such as liver transplantation, liver resection, or radiofrequency ablation. Patients with intermediate HCC are recommended transarterial chemoembolization (TACE) as the first-line treatment, according to the European Association for the Study of the Liver (EASL) because it can extend the two-year survival rate of patients [3, 4]. However, studies have shown that patients with advanced HCC could also get survival benefits from TACE [5-7]. For patients with advanced HCC, the SHARP trial showed that the median overall survival (OS) of patients with advanced HCC who received sorafenib was 2.8 months longer than that of patients who received a placebo [8]. Sorafenib is therefore 


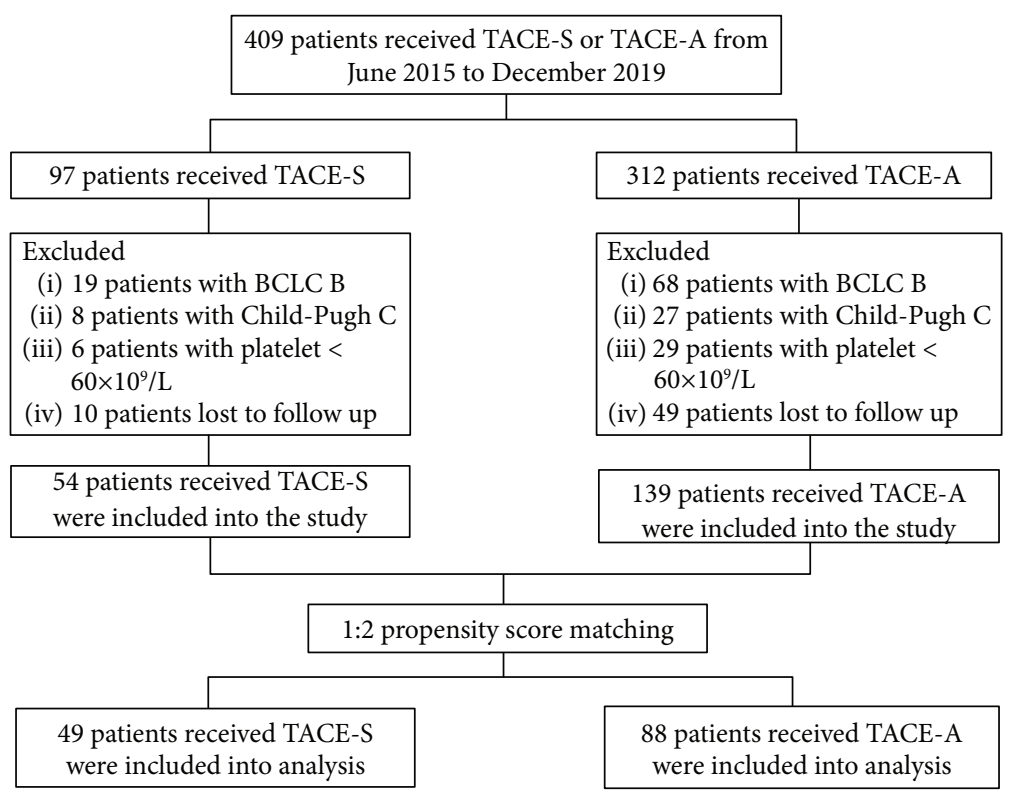

Figure 1: Flowchart of patient selection.

recommended as the first-line treatment for patients with advanced HCC. However, some patients did not see obvious survival benefits from sorafenib alone due to the low treatment response rate. Thus, sorafenib combined with other treatments can be used in the treatment of patients with advanced HCC. The most widely used combination treatment is TACE combined with sorafenib (TACE-S) which has been confirmed to be better than monotherapy [9-11]. Besides the limited efficacy, sorafenib was also a huge economic burden because of the high price for many patients with advanced HCC in China. Thus, cheaper and effective alternatives to sorafenib are needed.

Apatinib is an antiangiogenic drug that targets the vascular endothelial growth factor receptor-2 (VEGFR-2) and exerts similar antitumor effects to sorafenib.

Apatinib can target VEGF-R-2, inhibit the activation of VEGFR-2, and then block its downstream signal by specifically competing for adenosine triphosphate binding site in the cell. It can also inhibit the proliferation migration and the tube formation of human umbilical vein endothelial cells, blocking the generation of aortic rings $[12,13]$. In China, apatinib was approved for use in the treatment of gastric cancer and showed good efficacy $[14,15]$. Recently, studies have shown that patients with unresectable HCC could get survival benefits from apatinib, especially apatinib combined with TACE (TACE-A) [16-18].

Research has shown that patients treated with TACE-S or TACE-A had better survival benefits than patients treated with monotherapy (TACE or sorafenib) $[10,19,20]$. However, there is still a lack of evidence about whether patients with TACE-S had similar survival benefits to patients treated with TACE-A. Thus, we conducted a study to compare the efficacy and safety of TACE-S and TACE-A in patients with advanced HCC.

\section{Materials and Methods}

2.1. Patient Selection. We retrospectively reviewed the medical records of 409 consecutive patients with advanced HCC who received TACE-S or TACE-A from June 2015 to December 2019 in our institution. Before the initial TACE procedure, TACE-S was firstly recommended. For patients who refused to receive sorafenib, TACE-A was recommended.

The inclusion criteria of the study were as follows: (1) patients were diagnosed as advanced HCC based on the EASL guideline; (2) the Child-Pugh score of patients was A or B; (3) the Eastern Cooperative Oncology Group of patients was 0,1 , or 2 ; and (4) the platelet counts of patients were higher than $60 \times 10^{9} / \mathrm{L}$. The exclusion criteria were as follows: (1) patients received TACE or sorafenib or apatinib before they were included in the study; (2) patients with tumors that were metastasized from other organs; and (3) patients with diffuse tumors which could not be evaluated (Figure 1). This study was conducted in accordance with the Declaration of Helsinki and approved by the institutional review board of the institution. Informed consent was waived by the board.

\subsection{Treatments}

2.2.1. Transarterial Chemoembolization Procedure. TACE procedures were performed by three experienced radiologists with a minimum of 10 years of experience in interventional therapy. A $5 \mathrm{~F}$ catheter (Cook, Bloomington, IN, USA) was inserted into the hepatic artery. Then, a 3F microcatheter (Progreat, Terumo, Tokyo, Japan) was used to insert the tumor-feeding arteries. Subsequently, lipiodol (Lipiodol Ultrafluido, Guerbet, Villepinte, France) was mixed with doxorubicin hydrochloride to create an emulsion at the ratio of $1 \mathrm{~mL} / 2 \mathrm{mg}$. Depending on the size of the tumor and liver 
function, 5-20 mL emulsion was injected slowly into the tumor-feeding arteries until stasis of the feeding arteries of the tumor. If it was necessary, supplement embolization was performed using gelatin sponge particles (300-700 um, Cook, USA).

2.2.2. Sorafenib and Apatinib Administration. In the TACE$S$ group, sorafenib was orally and continuously administered $400 \mathrm{mg}$ twice daily when TACE was performed. In the TACE-A group, apatinib was orally taken 3-5 days after each TACE procedure at the starting dose of $500 \mathrm{mg} /$ day. The dose adjustments were based on the tolerance to the drug. The grading of adverse events associated with apatinib and sorafenib was conducted according to the National Cancer Institute Common Terminology Criteria for Adverse Events (version 4.0). In the case of grade 3 or 4 apatinib- or sorafenib-related adverse events, the dose of apatinib was modified to $250 \mathrm{mg} /$ day and the dose of sorafenib was adjusted to $400 \mathrm{mg}$ once daily until the adverse effects were alleviated.

2.3. Endpoints. The primary endpoint of the study was overall survival (OS), which was defined as the time from the first TACE to patients' death or the end of the study. The secondary endpoints were progression-free survival (PFS) and disease control rate (DCR) 3 months after the initial TACE. The PFS was defined as the time from the initial TACE to the progression of the tumor, the time of patients' death, or the time of the end of the study. The DCR was defined as the proportion of patients with complete response $(\mathrm{CR})$, partial response (PR), and stable response (SD) in patients with TACE-S or TACE-A based on the mRECIST criteria [21].

2.4. Follow-Up. All patients who were included in the study were followed up. In the study, patients were required to receive CT or MRI and laboratory tests every 4-6 weeks after the initial TACE for 6 months. After 6 months, patients were required to receive CT or MRI and laboratory tests every 23 months. The images of patients were reviewed by two radiologists (one with 24 years of radiographic experience and one with 20 years of radiographic experience) who were blinded to the treatment of the patients. If the results of their evaluation were different, another radiologist (with 31 years of radiographic experience) evaluated the results and gave the final results. When the CT or MRI image showed that the tumor remained or the tumor progressed, another TACE was recommended to these patients. The follow-up end time of the study was July $30,2020$.

2.5. Statistical Analysis. Continuous variables were compared by independent sample $t$-test or Mann-Whitney $U$ test. Categorical variables were compared by the Chi-square test or Fisher's test between the two groups. Kaplan-Meier was used to plot the survival curves and the survival benefits in both groups and compared by log-rank. Adjusted Cox regression risk model was used to predict the potential factors which might influence the OS and PFS. Propensity score matching (PSM) was used to reduce selection bias. All characteristics were included in PSM analysis, and $1: 2$ ratio matching with an optimal caliper of 0.2 without replacement generated 139 patients (49 patients with TACE-S and 88 patients with TACE-A). The Landmark method was used in the study because it determines survival based on response status assessed at each specific time point. Because the Landmark is recommended in oncology guidelines for TACE-S/TACE-A analysis, a 12-month Landmark for OS and PFS was used in the study before and after PSM. All statistical analysis was conducted by SPSS 24.0 (IBM Corp, Armonk, NY, USA) and R 3.6.2.

\section{Results}

3.1. Characteristics of Patients. In this study, a total of 193 patients with advanced HCC who received TACE-S or TACE-A were included in the analysis. Among them, 54 patients received TACE-S and 139 patients received TACEA. The median time of follow-up was 11.5 months (range, 2.5-58.1 months) in the TACE-S group and 11.5 months (range, 1-59.2 months) in the TACE-A group. In the TACES group, 43 patients of the 54 died and 120 patients of 139 patients died in the TACE-A group during the follow-up (Table 1).

3.2. Survival Outcomes and Tumor Response. Before PSM, the mOS was 12.4 months (95\%CI: 10.7-14.1 months) in the TACE-S group and 11.5 months (95\%CI: $8.8-14.2$ months) in the TACE-A group $(P=0.703)$. The mPFS was 5.7 months (95\%CI: 5.0-6.4 months) in the TACE-S group and 5 months (95\%CI: 3.8-6.2 months) in the TACE-A group $(P=0.514)$ (Figure 2). The DCR in the previous 3 months was $56.8 \%(79 / 139)$ in the TACE-A group and $64.8 \%(35 / 54)$ in the TACE-S group $(P=0.311)$. After PSM, the mOS and mPFS were 12.2 months (95\%CI: 8.9-15.5 months) and 5.4 months (95\%CI: 4.4-6.4 months) in the TACE-S group and 11.9 months (95\%CI: 10.2-13.6 months), and 5.1 months (95\%CI: 4.0-6.2 months) in the TACE-A group $(P=0.672$ and $P=0.808)$ (Figure 3 ). The DCR was $60.2 \%(53 / 88)$ in the TACE-A and $63.3 \%$ (31/ $49)$ in the TACE-S group $(P=0.726)$.

3.3. Landmark Analysis and Cox Regression Analysis. The Landmark analysis showed that TACE-A did not increase the mortality risk (HR: 1.255, 95\%CI: 0.796-1.978, $P=0.329$ ) or tumor recurrence risk (HR: $1.054,95 \% \mathrm{CI}$ : $0.744-1.493, P=0.767)$ compared to TACE-S in the previous 12 months. After 12 months, TACE-A also did not increase the mortality risk (HR: $0.832,95 \%$ CI: $0.482-1.436$, $P=0.508$ ) or tumor recurrence risk (HR: $1.730,95 \% \mathrm{CI}$ : 0.592-5.049, $P=0.316$ ) compared to TACE-S (Figure 2). Similar results were presented after PSM. TACE-A did not increase the mortality risk (HR: $1.031,95 \%$ CI: $0.624-1.703$, $P=0.904$ ) or tumor recurrence risk (HR: $0.984,95 \% \mathrm{CI}$ : $0.671-1.443, P=0.935)$ compared to TACE-S in the previous 12 months. After 12 months, TACE-A also did not 
TABLE 1: Baseline characteristics of patients with TACE-S and TACE-A before PSM and after PSM.

\begin{tabular}{|c|c|c|c|c|c|c|}
\hline \multirow[b]{2}{*}{ Characteristics } & \multicolumn{3}{|c|}{ Before matching } & \multicolumn{3}{|c|}{ After matching } \\
\hline & $\begin{array}{c}\text { TACE-S }(\text { mean } \pm \text { SD }) \\
(N=54 / \%)\end{array}$ & $\begin{array}{c}\text { TACE-A }(\text { mean } \pm \text { SD }) \\
(N=139 / \%)\end{array}$ & $\begin{array}{c}P \\
\text { value }\end{array}$ & $\begin{array}{c}\text { TACE-S }(\text { mean } \pm \text { SD }) \\
(N=49 / \%)\end{array}$ & $\begin{array}{c}\text { TACE-A (mean } \pm \text { SD }) \\
(N=88 / \%)\end{array}$ & $\begin{array}{c}P \\
\text { value }\end{array}$ \\
\hline Age & $53.1 \pm 10.6$ & $51.2 \pm 9.8$ & 0.243 & $52.8 \pm 10.7$ & $51.8 \pm 8.8$ & 0.593 \\
\hline ALT & $57.5 \pm 78.3$ & $60.3 \pm 69.0$ & 0.808 & $57.9 \pm 82.0$ & $51.2 \pm 57.5$ & 0.579 \\
\hline AST & $73.8 \pm 87.5$ & $78.2 \pm 147.1$ & 0.839 & $76.6 \pm 91.5$ & $52.9 \pm 45.4$ & $0.194 \mathrm{a}$ \\
\hline Hemoglobin & $128.2 \pm 21.6$ & $126.4 \pm 20.2$ & 0.588 & $127.7 \pm 22.5$ & $125.2 \pm 21.2$ & 0.515 \\
\hline Platelet & $155.6 \pm 68.7$ & $164.5 \pm 80.7$ & 0.475 & $157.0 \pm 71.4$ & $163.0 \pm 78.2$ & 0.660 \\
\hline Lymphocytes & $1.3 \pm 0.7$ & $1.2 \pm 0.5$ & 0.396 & $1.2 \pm 0.6$ & $1.2 \pm 0.6$ & 0.695 \\
\hline Neutrophils & $3.4 \pm 1.9$ & $3.7 \pm 1.9$ & 0.363 & $3.6 \pm 1.9$ & $3.6 \pm 1.8$ & 0.927 \\
\hline Tumor size & $8.2 \pm 4.4$ & $9.0 \pm 4.3$ & 0.278 & $8.5 \pm 4.5$ & $8.1 \pm 4.0$ & 0.589 \\
\hline \multicolumn{7}{|l|}{ Gender } \\
\hline Male & $51(94.4)$ & $119(85.6)$ & \multirow{2}{*}{0.089} & $46(93.9)$ & $80(90.9)$ & \multirow{2}{*}{0.776} \\
\hline Female & $3(5.6)$ & $20(14.4)$ & & $3(6.1)$ & $8(9.1)$ & \\
\hline \multicolumn{7}{|l|}{ HBV infection } \\
\hline Yes & $41(75.9)$ & $108(77.7)$ & \multirow{2}{*}{0.792} & $39(79.6)$ & $63(71.6)$ & \multirow{2}{*}{0.303} \\
\hline No & $13(24.1)$ & $31(22.3)$ & & $10(20.4)$ & $25(28.4)$ & \\
\hline \multicolumn{7}{|l|}{ AFP } \\
\hline$<200$ & $30(55.6)$ & $55(39.6)$ & \multirow{2}{*}{0.045} & $25(51)$ & $42(47.7)$ & \multirow{2}{*}{0.712} \\
\hline$\geq 200$ & $24(44.4)$ & $84(60.4)$ & & $24(49)$ & $46(52.3)$ & \\
\hline \multicolumn{7}{|l|}{ TACE session } \\
\hline 1 & $11(20.4)$ & $24(17.3)$ & \multirow{2}{*}{0.615} & $10(20.4)$ & $14(15.9)$ & \multirow{2}{*}{0.507} \\
\hline$\geq 2$ & $43(79.6)$ & $115(82.7)$ & & $39(79.6)$ & $74(84.1)$ & \\
\hline \multicolumn{7}{|l|}{ Tumor number } \\
\hline 1 & $15(27.8)$ & $43(30.9)$ & \multirow{2}{*}{0.688} & $14(28.6)$ & $24(27.3)$ & \multirow{2}{*}{0.871} \\
\hline$\geq 2$ & $39(72.2)$ & $96(69.1)$ & & $35(71.4)$ & $64(72.7)$ & \\
\hline \multicolumn{7}{|l|}{ Portal invasion } \\
\hline Yes & $31(57.4)$ & $84(60.4)$ & \multirow{2}{*}{0.701} & $28(57.1)$ & $46(52.3)$ & \multirow{2}{*}{0.584} \\
\hline No & $23(42.6)$ & $55(39.6)$ & & $21(42.9)$ & $42(47.7)$ & \\
\hline \multicolumn{7}{|c|}{ Extrahepatic metastasis } \\
\hline Yes & $39(72.2)$ & $100(71.9)$ & \multirow{2}{*}{0.969} & $36(73.5)$ & $67(76.1)$ & \multirow{2}{*}{0.729} \\
\hline No & $15(27.8)$ & $39(28.1)$ & & $13(26.5)$ & $21(23.9)$ & \\
\hline \multicolumn{7}{|l|}{ Cirrhosis } \\
\hline Yes & $34(63)$ & $85(61.2)$ & 0816 & $31(63.3)$ & $53(60.2)$ & 0726 \\
\hline No & $20(37)$ & $54(38.8)$ & 0.816 & $18(27.7)$ & $35(39.8)$ & 0.720 \\
\hline Child-Pugh & & & & & & \\
\hline A & $23(42.6)$ & $66(47.5)$ & & $21(42.9)$ & $42(47.7)$ & \\
\hline B & $31(57,4)$ & $73(52.5)$ & 0.541 & $28(57.1)$ & $46(52.3)$ & 0.584 \\
\hline ECOG & & & & & & \\
\hline 0 & $18(33.3)$ & $22(15.8)$ & & $14(28.6)$ & $21(23.9)$ & \\
\hline 1 & $29(53.7)$ & $87(62.6)$ & 0.021 & $28(57.1)$ & $57(64.8)$ & 0.675 \\
\hline 2 & $7(13)$ & $30(21.6)$ & & $7(14,3)$ & $10(11.3)$ & \\
\hline
\end{tabular}

Abbreviations: TACE-S: transarterial chemoembolization combined with sorafenib; TACE-A: transarterial chemoembolization combined with apatinib; ALT: alanine aminotransferase; AST: aspartate aminotransferase; AFP: alpha-fetoprotein; ECOG: Eastern Cooperative Oncology Group.

increase the mortality risk (HR: $0.780,95 \% \mathrm{CI}: 0.430-1.413$, $P=0.413)$ or tumor recurrence risk (HR: $1.825,95 \% \mathrm{CI}$ : 0.514-6.477, $P=0.352$ ) compared to TACE-S (Figure 3). In the Cox regression analysis, after adjustment for age, ALT, AST, hemoglobin, platelet, lymphocytes, neutrophils, tumor size, gender, HBV infection, AFP level, TACE session, tumor number, portal invasion, extrahepatic metastases, cirrhosis, Child-Pugh score, and Eastern Cooperative Oncology (ECOG), TACE-A did not increase the mortality risk (HR: $0.908,95 \% \mathrm{CI}$ : $0.620-1.330, P=0.620$ ) or tumor recurrence risk (HR: 0.906, 95\%CI: 0.634-1.295, $P=0.589$ ) compared to TACE-S before PSM (Table 2). In the Landmark analysis for 12 months, TACE-A did not increase the mortality risk (HR: $1.086,95 \% \mathrm{CI}$ : 0.663-1.780, $P=0.744)$ or tumor recurrence risk (HR: $0.906,95 \% \mathrm{CI}$ :
0.624-1.314, $P=0.601)$ compared to TACE-S. After 12 months, TACE-A did not increase the mortality risk (HR: $0.627,95 \% \mathrm{CI}: 0.307-1.281, P=0.200$ ) but reduced the tumor recurrence risk (HR: 0.044, 95\%CI: 0.002-0.810, $P=0.036)$ compared to TACE-S (Table 2).

3.4. Subgroup Analysis. In the adjusted Cox regression analysis, after adjustment for age, ALT, AST, hemoglobin, platelet, lymphocytes, neutrophils, tumor size, gender, HBV infection, AFP level, TACE session, tumor number, cirrhosis, and ECOG, TACE-A did not increase the mortality risk (Child-Pugh A: HR: 0.590, 95\%CI: 0.305-1.140, $P=0.116$; Child-Pugh B: HR: 1.056, 95\%CI: $0.612-1.824, P=0.844$ ) or tumor recurrence risk (Child- 

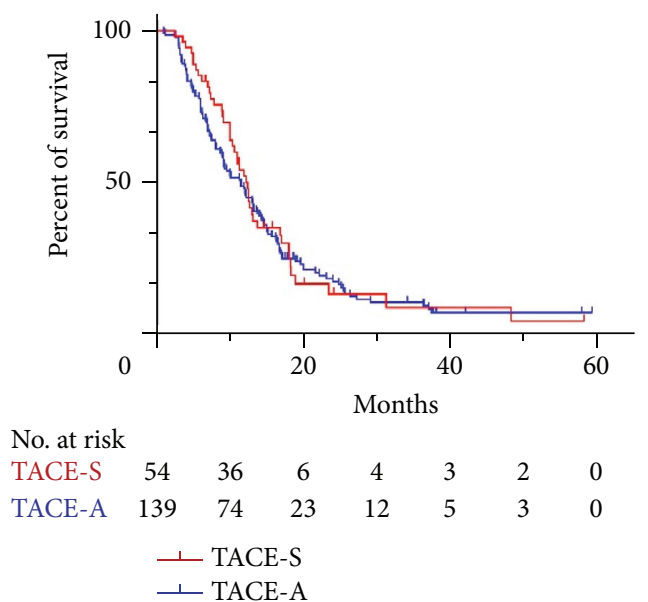

(a)

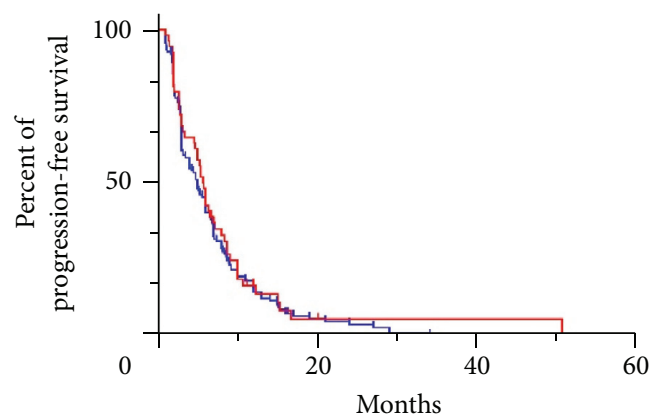

\begin{tabular}{|c|c|c|c|c|c|c|c|}
\hline \multirow[b]{2}{*}{ No. at risk } & \multicolumn{7}{|c|}{ 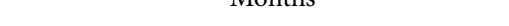 } \\
\hline & & & & & & & \\
\hline TACE-S & 54 & 12 & 2 & 1 & 1 & 1 & ( \\
\hline TACE-A & 139 & 19 & 7 & 2 & 0 & 0 & . \\
\hline
\end{tabular}

(c)
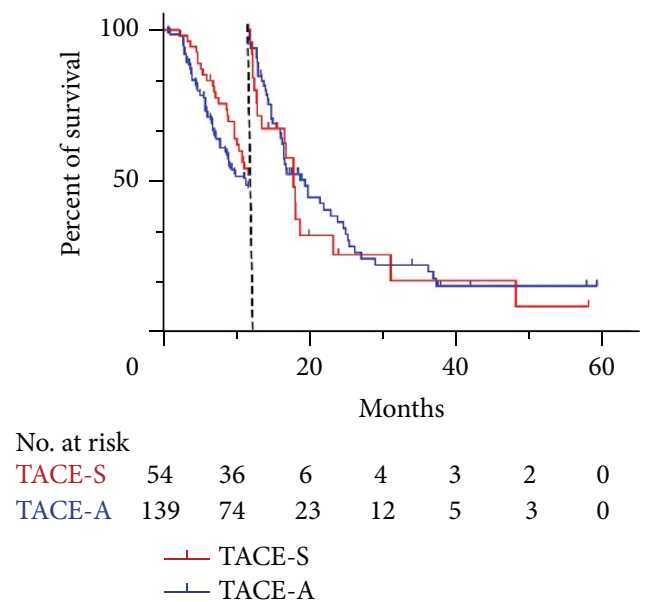

(b)

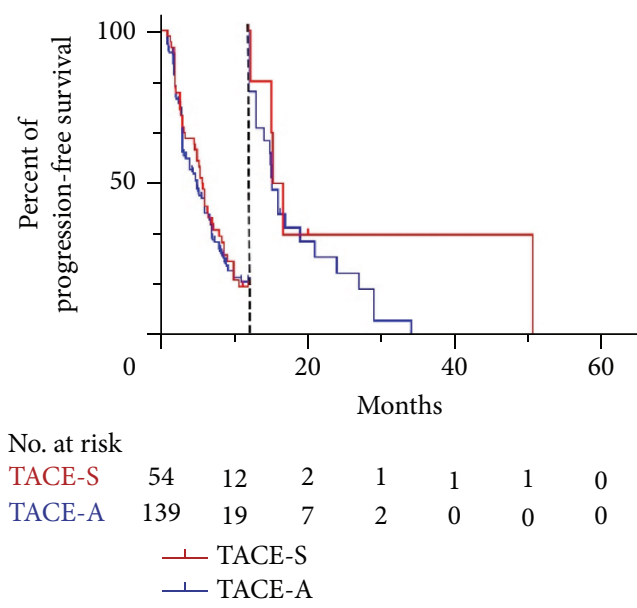

(d)

\begin{tabular}{lcccr}
\hline \multirow{2}{*}{ Statistical analysis } & \multicolumn{2}{c}{ Overall survival } & \multicolumn{2}{c}{ Progression-free survival } \\
\cline { 2 - 5 } & HR $(95 \%$ CI $)$ & P value & HR $(95 \% \mathrm{CI})$ & P value \\
\hline $\begin{array}{l}\text { Overall (TACE-A/TACE-S) } \\
\text { Previous 12 months landmark }\end{array}$ & $1.070(0.754,1.519)$ & 0.705 & $1.113(0.800,1.547)$ & 0.525 \\
$\begin{array}{l}\text { TACE-A/TACE-S) } \\
\begin{array}{l}\text { After 12 months landmark } \\
\text { (TACE-A/TACE-S) }\end{array}\end{array}$ & $0.253(0.796,1.978)$ & 0.508 & $1.054(0.744,1.493)$ & 0.767 \\
\hline
\end{tabular}

FIGURE 2: Kaplan-Meier curves and landmark analysis of OS and PFS before PSM. (a) Kaplan-Meier curve of OS. (b) Kaplan-Meier curve for landmark analysis of OS. (c) Kaplan-Meier curve of PFS. (d) Kaplan-Meier curve for landmark analysis of PFS. (Table) Univariable Cox regression analysis for OS and PFS.

Pugh A: HR: 0.631, 95\%CI: 0.349-1.140, $P=0.127$; ChildPugh B: HR: 1.066, 95\%CI: 0.648-1.754, $P=0.802$ ) compared to TACE-S in the patients with Child-Pugh A and B scores. Similarly, TACE-A did not increase the mortality risk (with extrahepatic metastases: HR: 0.854, 95\%CI: 0.534-1.365, $P=0.510$; without extrahepatic metastases: HR: $0.948,95 \% \mathrm{CI}: 0.352-2.559, P=0.917)$ or tumor recurrence risk (with extrahepatic metastases: HR: 1.018, 95\% CI: $0.653-1.588, P=0.936$; without extrahepatic metastases: HR: $0.586,95 \% \mathrm{CI}: 0.226-1.521, P=0.272$ ) compared to
TACE-S in the patients with extrahepatic metastases and without extrahepatic metastases. Again, TACE-A also did not increase mortality risk (with portal invasion: HR: 0.967, 95\%CI: $0.555-1.683, P=0.905$; without portal invasion: HR: 0.928 , 95\%CI: $0.479-1.799, P=0.825$ ) or tumor recurrence risk (with portal invasion: HR: 0.851, 95\%CI: 0.508-1.426, $P=0.541$; without portal invasion: HR: 0.995 , 95\%CI: $0.515-1.925, P=0.989)$ compared with TACE-S in the patients with portal invasion and without portal invasion before PSM (Figure 4). 


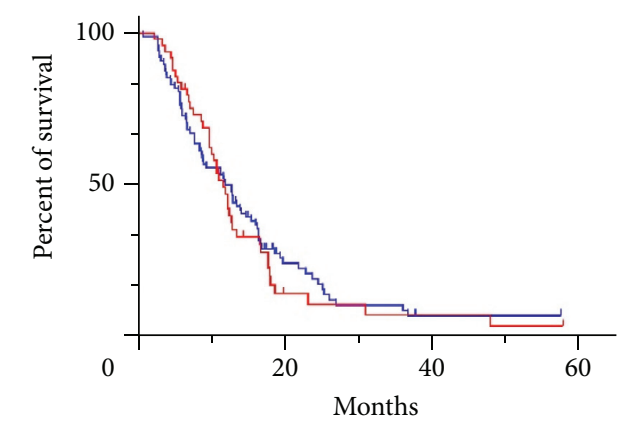

No. at risk

$\begin{array}{llllllll}\text { TACE-S } & 49 & 32 & 6 & 4 & 3 & 2 & 0\end{array}$

$\begin{array}{llllllll}\text { TACE-A } & 88 & 50 & 16 & 7 & 3 & 2 & 0\end{array}$

$\longrightarrow$ TACE-S

$\longrightarrow$ TACE-A

(a)

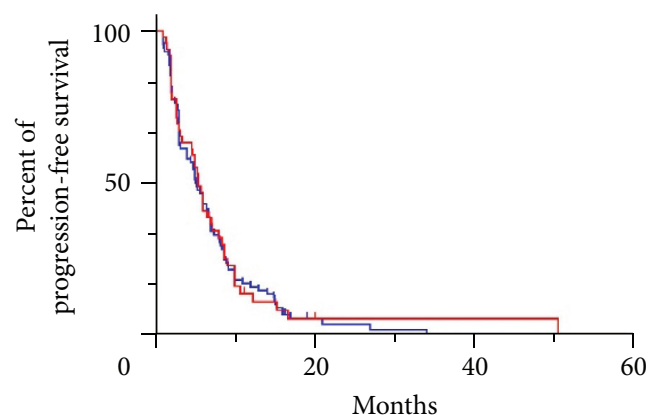

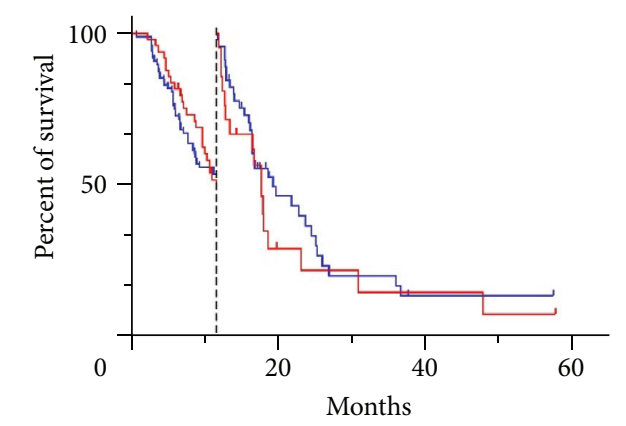

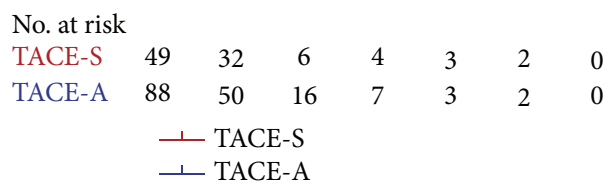

(b)
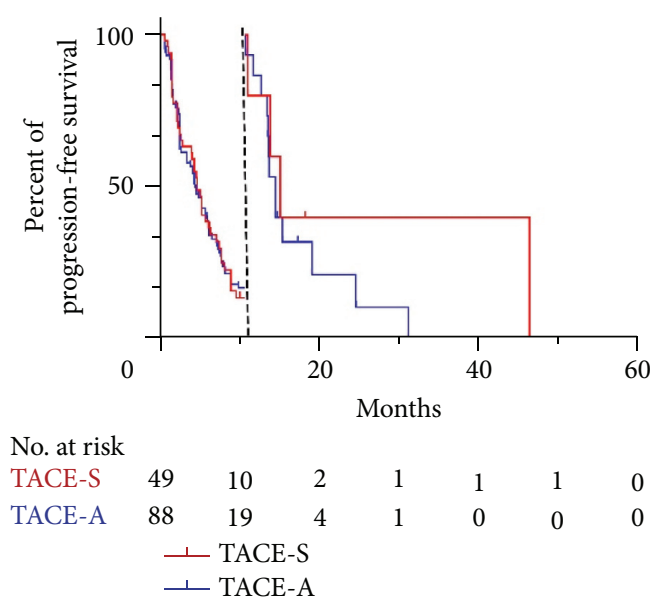

(d)

(c)

Progression-free survival

\begin{tabular}{lcccc}
\hline \multirow{2}{*}{ Statistical analysis } & \multicolumn{2}{c}{ Overall survival } & \multicolumn{2}{c}{ Progression-free survival } \\
\cline { 2 - 5 } & HR (95\%CI) & P value & HR $(95 \%$ CI $)$ & P value \\
\hline $\begin{array}{l}\text { Overall (TACE-A/TACE-S) } \\
\text { Previous 12 months landmark }\end{array}$ & $0.921(0.627,1.353)$ & 0.674 & $1.045(0.726,1.505)$ & 0.812 \\
$\begin{array}{l}\text { (TACE-A/TACE-S) } \\
\begin{array}{l}\text { After 12 months landmark } \\
\text { (TACE-A/TACE-S) }\end{array}\end{array}$ & $0.780(0.430,1.413)$ & 0.508 & $1.825(0.514,6.477)$ & 0.352 \\
\hline
\end{tabular}

FIGURE 3: Kaplan-Meier curves and landmark analysis of OS and PFS after PSM. (a) Kaplan-Meier curve of OS.(b) Kaplan-Meier curve for landmark analysis of OS. (c) Kaplan-Meier curve of PFS. (d) Kaplan-Meier curve for landmark analysis of PFS. (Table) Univariable Cox regression analysis for OS and PFS.

3.5. Safety of Patients with TACE-S or TACE-S. In the study, the adverse events of patients with TACE and sorafenib and apatinib were evaluated. For all grades of adverse events, there was no statistically significant difference of fever $(P=0.216)$, abdominal pain $(P=0.886)$, nausea $(P=0.443)$, vomiting $(P=0.235)$, anorexia $(P=0.692)$, diarrhea $(P=0.749)$, hypertension $(P=0.085)$, fatigue $(P=0.343)$, hand-foot reaction $(P=0.715)$, gastrointestinal hemorrhage $(P>0.999)$, headache $(P=0.921)$, and proteinuria $(P=0.892)$ in the TACE-S group and TACE-A group. Similarly, there was no statistically significant difference of relative adverse events (grades $\geq 3$ ) between the two groups (all $P>0.05$ ) (Table 3 ).

\section{Discussion}

Although the SHARP and ORIENTAL clinical trials showed that patients with advanced HCC who received sorafenib had better survival benefits than a placebo group, the response rate of patients who received sorafenib was still low $[8,22-24]$. Thus, TACE combined with sorafenib was used in the treatment of advanced HCC more widely and showed 
TABLE 2: Adjusted Cox regression for OS and PFS before PSM, adjusted for age, ALT, AST, hemoglobin, platelet, lymphocytes, neutrophils, tumor size, gender, HBV infection, AFP level, TACE session, tumor number, portal invasion, extrahepatic metastases, cirrhosis, Child-Pugh, and ECOG.

\begin{tabular}{lccc}
\hline Characteristics & Overall survival & Progression-free survival \\
Overall & HR $(95 \% \mathrm{CI})$ & $P$ value & HR $(95 \% \mathrm{CI})$ \\
$\begin{array}{l}\text { TACE-S } \\
\text { TACE-A }\end{array}$ & Reference & Reference \\
\hline $\begin{array}{l}\text { Before 12-month Landmark } \\
\text { TACE-S }\end{array}$ & $0.908(0.620,1.330)$ & 0.620 & $0.906(0.634,1.295)$ \\
TACE-A & Reference & & 0.589 \\
\hline $\begin{array}{l}\text { Before 12-month Landmark } \\
\text { TACE-S }\end{array}$ & Reference & 0.744 & $0.906(0.624,1.314)$ \\
TACE-A & $0.627(0.307,1.281)$ & & 0.601 \\
\hline
\end{tabular}

Abbreviations: TACE-S: transarterial chemoembolization combined with sorafenib; TACE-A: transarterial chemoembolization combined with apatinib.

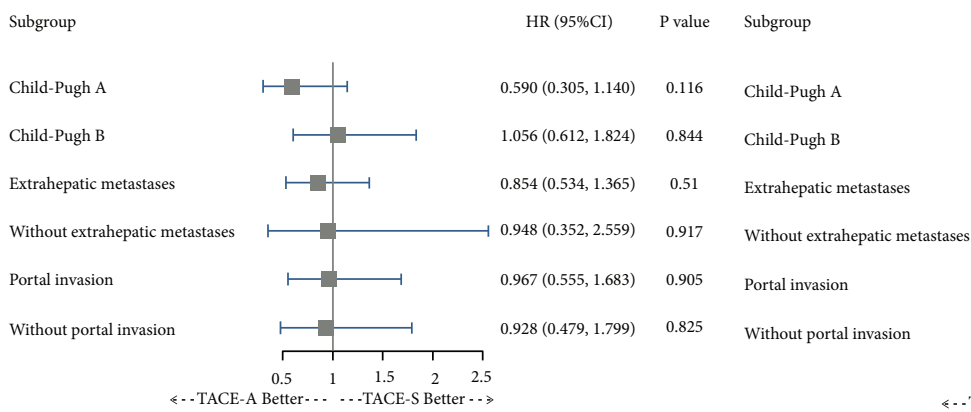

(a)

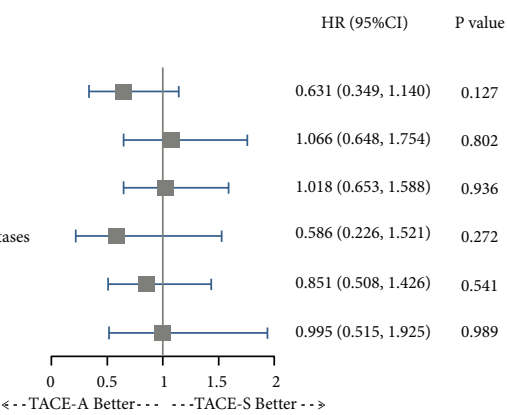

(b)

FIGURE 4: Adjusted Cox regression analysis for subgroups analysis before PSM, adjusted for age, ALT, AST, HB, PLT, LYM, NEU, size, gender, HBV, APF, TACE session, tumor number, cirrhosis, and ECOG. (a) Subgroups analysis for OS. (b) Subgroup analysis for PFS.

TABLE 3: Adverse events of patients after receiving TACE-S and TACE-A before PSM.

\begin{tabular}{|c|c|c|c|c|c|c|}
\hline \multirow{2}{*}{ Adverse events } & \multicolumn{3}{|c|}{ All grades } & \multicolumn{3}{|c|}{ Grades $\geq 3$} \\
\hline & TACE-S & TACE-A & $P$ value & TACE-S & TACE-A & $P$ value \\
\hline Fever & $23(42.6)$ & $73(52.5)$ & 0.216 & $1(1.9)$ & $2(3.7)$ & $>0.999$ \\
\hline Abdominal pain & $44(81.5)$ & $112(80.6)$ & 0.886 & $2(3.7)$ & $5(3.6)$ & 0.972 \\
\hline Nausea & $35(64.8)$ & $98(70.5)$ & 0.443 & $1(1.9)$ & $4(2.9)$ & 0.677 \\
\hline Vomiting & $24(44.4)$ & $75(54)$ & 0.235 & $1(1.9)$ & $3(2.2)$ & 0.892 \\
\hline Anorexia & $22(40.7)$ & $61(46.1)$ & 0.692 & $0(0)$ & $0(0)$ & $>0.999$ \\
\hline Diarrhea & $12(22.2)$ & $28(20.1)$ & 0.749 & $0(0)$ & $0(0)$ & $>0.999$ \\
\hline Hypertension & $19(35.2)$ & $68(48.9)$ & 0.085 & $1(1.9)$ & $2(1.4)$ & $>0.999$ \\
\hline Fatigue & $6(11.1)$ & $23(16.5)$ & 0.343 & $0(0)$ & $0(0)$ & $>0.999$ \\
\hline Hand-foot skin reaction & $7(13.0)$ & $21(15.1)$ & 0.704 & $2(3.7)$ & $5(3.6)$ & 0.687 \\
\hline Gastrointestinal hemorrhage & $0(0)$ & $2(1.4)$ & $>0.999$ & $0(0)$ & $0(0)$ & $>0.999$ \\
\hline Headache & $9(16.7)$ & $24(17.3)$ & 0.921 & $0(0)$ & $1(0.7)$ & $>0.999$ \\
\hline Proteinuria & $1(1.9)$ & $3(2.2)$ & 0.892 & $0(0)$ & $0(0)$ & $>0.999$ \\
\hline
\end{tabular}

Abbreviations: TACE-S: transarterial chemoembolization combined with sorafenib; TACE-A: transarterial chemoembolization combined with apatinib. 
good efficacy. However, due to the low response rate and high cost of sorafenib, another selective drug, apatinib, was used in the treatment of advanced HCC. Several studies have showed that patients treated with TACE-A had improved survival compared to patients with single treatment. However, any differences in efficacy between the patients with TACE-S and TACE-A were still unclear. Thus, the aim of the study was to compare the efficacy and the safety of advanced HCC patients treated with either TACE-S or TACE-A.

Previous studies have shown that the mOS of advanced HCC patients with TACE-S ranged from 11.2 months to 17.5 months, and the median time to progression (mTTP) or mPFS ranged from 4.3 months to 7.6 months [19, 20, 25-28]. The mOS of advanced HCC patients with TACE-A ranged from 12 to 22 months and mTTP from 6.1 to 9.5 months $[18,29,30]$. In the current study, we got similar results. The mOS and mPFS of patients with TACE-S were 12.4 months and 5.7 months, respectively. Compared with previous studies analyzing the survival of patients who received TACE plus sorafenib, the mOS and mPFS of the current study were shorter than the mOS (16.5 months and 17.5 months) and mPFS (7 months) or mTTP (7 months) in the two studies $[19,20]$. The reason might be the heterogeneity of the included patients. The current study included $57.4 \%$ of patients with Child-Pugh B and $72.2 \%$ of patients with extrahepatic metastases. However, the study conducted by Koch et al. included $26 \%$ of patients with Child-Pugh B and $41 \%$ of patients with extrahepatic spread. The study conducted by Kim et al. included $8 \%$ of patients with ChildPugh B. The mOS and mPFS of patients with TACE-A were 11.5 months and 5 months, respectively. Before and after PSM, there was no statistically significant difference of mOS and mPFS between the patients with TACE-S and the patients with TACE-A. Some patients with HCC who received TACE could realize complete embolization, which increases the hypoxia of tumor cells. The hypoxic microenvironment of tumor cells can make tumor cells generate proangiogenic factors, which leads to tumor angiogenesis and tumor progression. Apatinib is an antiangiogenic drug. It can prevent angiogenesis after patients receive TACE and prevent further tumor progression, which might be the reason why advanced HCC patients who received TACE-A had similar survival benefits to patients who received TACE-S.

In the study, the Landmark was used to mitigate guarantee-time bias, and a 12-month period was used $[31,32]$. The study results showed that patients with TACEA did not have an increased mortality risk or tumor recurrence compared with patients treated with TACE-S in the univariable regression and multivariable regression analysis at the previous 12 months and 12 months after PSM. The results indicate that the tumor response of patients who received TACE-A was similar to patients who received TACE-S at both early and late times after the initial TACE. In the adjusted Cox regression analysis for all patients, all factors were included in the analysis to reduce potential factors which might influence the overall survival and tumor progression. After reducing the potential influencing factors, TACE-A did not increase mortality risk or tumor recurrence relative to TACE-S. Previous studies showed that the liver function, tumor portal invasion, and distant metastases might influence the survival of patients with HCC. Thus, in the current study, the subgroups analysis divided patients into six groups, such as patients with Child-Pugh A score, patients with Child-Pugh B score, patients with portal invasion, patients without portal invasion, patients with tumor distant metastases, and patients without distant metastases. The adjusted Cox regression analysis showed that TACE-A did not increase mortality risk or tumor recurrence risk compared with TACE-S in different groups. The results of subgroups analysis indicated that the different statuses of patients with advanced HCC who received TACE-A could still get similar survival benefits compared with patients who received TACE-S.

In the study, the adverse events related to TACE or apatinib or sorafenib were evaluated. The III and IV grades of adverse events in the TACE-A treatment group were not higher than the TACE-S treatment group. And after reducing the dose administration of apatinib, the adverse events of patients with TACE-A and TACE-S were relieved. The results of this study showed that apatinib is a welltolerated treatment option with an acceptable safety profile for patients with advanced HCC compared with sorafenib.

Some limitations existed in the study. Firstly, the study was a retrospective study, which led to the existence of selection bias. However, the PSM was used in the study to reduce selection bias. Secondly, the number of patients enrolled in the study was small, especially the sample of patients with TACE-S, which might influence the accuracy of the conclusion. Finally, the selection of drugs might be influenced by the patients' willingness, which might result in some patients receiving suboptimal treatments. However, until now, there have been no criteria indicating which kind of advanced HCC patients are suitable for sorafenib or apatinib. Consequently, more studies are needed to confirm the results of the study.

\section{Conclusion}

The study showed that patients with advanced HCC who received TACE-A had comparable survival benefits to patients who received TACE-S. TACE-A might be a good choice for patients who were unwilling to receive TACE-S.

\section{Abbreviations}

TACE- Transarterial chemoembolization combined with S: $\quad$ sorafenib

TACE- Transarterial chemoembolization combined with A: apatinib

HCC: Hepatocellular carcinoma

BCLC: Barcelona clinic liver cancer

OS: Overall survival

PFS: Progression-free survival

DCR: Disease control rate

PSM: Propensity score matching

ECOG: Eastern cooperative oncology group

AFP: Alpha-fetoprotein. 


\section{Data Availability}

The data used in the study are available from the corresponding authors on reasonable request.

\section{Ethical Approval}

This study was carried out in compliance with the Helsinki Declaration. The study was approved by the Ethics Committee Board of Tongji Medical College, Huazhong University of Science and Technology.

\section{Consent}

The requirement of informed consent was obtained from the patients.

\section{Conflicts of Interest}

The authors declare that they have no conflicts of interest.

\section{Authors' Contributions}

$\mathrm{CZ}$ and $\mathrm{BL}$ conceptualized and designed the study. $\mathrm{CZ}$ provided administrative support. LC and TS did data collection. LC, LW, DX, and ST did data analysis. WZ, LW, YR, and $\mathrm{LC}$ wrote the manuscript. $\mathrm{CZ}$ and $\mathrm{BL}$ reviewed the manuscript. All authors approved the final manucript. LC, TS, and LW contributed equally to the manuscript.

\section{Acknowledgments}

This study was supported by the National Natural Science Foundation of China (no. 81873919).

\section{References}

[1] F. Bray, J. Ferlay, I. Soerjomataram, R. L. Siegel, L. A. Torre, and A. Jemal, "Global cancer statistics 2018: GLOBOCAN estimates of incidence and mortality worldwide for 36 cancers in 185 countries," CA: A Cancer Journal for Clinicians, vol. 68, no. 6, pp. 394-424, 2018.

[2] P. C. Valery, M. Laversanne, P. J. Clark, J. L. Petrick, K. A. McGlynn, and F. Bray, "Projections of primary liver cancer to 2030 in 30 countries worldwide," Hepatology, vol. 67, no. 2, pp. 600-611, 2018.

[3] EASL Clinical Practice Guidelines, "Management of hepatocellular carcinoma," Journal of Hepatology, vol. 69, no. 1, pp. 182-236, 2018.

[4] J. Llovet and J. Bruix, "Systematic review of randomized trials for unresectable hepatocellular carcinoma: chemoembolization improves survival," Hepatology, vol. 37, no. 2, pp. 429-442, 2003

[5] Z.-J. Niu, Y.-L. Ma, P. Kang et al., "Transarterial chemoembolization compared with conservative treatment for advanced hepatocellular carcinoma with portal vein tumor thrombus: using a new classification," Medical Oncology, vol. 29, no. 4, pp. 2992-2997, 2012.

[6] J. Luo, R.-P. Guo, E. C. H. Lai et al., "Transarterial chemoembolization for unresectable hepatocellular carcinoma with portal vein tumor thrombosis: a prospective comparative study," Annals of Surgical Oncology, vol. 18, no. 2, pp. 413-420, 2011.

[7] T.-C. Xue, X.-Y. Xie, L. Zhang, X. Yin, B.-H. Zhang, and Z.-G. Ren, "Transarterial chemoembolization for hepatocellular carcinoma with portal vein tumor thrombus: a metaanalysis," BMC Gastroenterology, vol. 13, no. 1, p. 60, 2013.

[8] J. M. Llovet, S. Ricci, V. Mazzaferro et al., "Sorafenib in advanced hepatocellular carcinoma," New England Journal of Medicine, vol. 359, no. 4, pp. 378-390, 2008.

[9] X. Zhang, K. Wang, M. Wang et al., "Transarterial chemoembolization (TACE) combined with sorafenib versus TACE for hepatocellular carcinoma with portal vein tumor thrombus: a systematic review and meta-analysis," Oncotarget, vol. 8, no. 17, pp. 29416-29427, 2017.

[10] M. Kudo, K. Ueshima, M. Ikeda et al., "Randomised, multicentre prospective trial of transarterial chemoembolisation (TACE) plus sorafenib as compared with TACE alone in patients with hepatocellular carcinoma: TACTICS trial," Gut, vol. 69, no. 8, pp. 1492-1501, 2020.

[11] J.-F. Geschwind, M. Kudo, J. A. Marrero et al., "TACE treatment in patients with sorafenib-treated unresectable hepatocellular carcinoma in clinical practice: final analysis of GIDEON,” Radiology, vol. 279, no. 2, pp. 630-640, 2016.

[12] S. Tian, H. Quan, C. Xie et al., "YN968D1 is a novel and selective inhibitor of vascular endothelial growth factor receptor-2 tyrosine kinase with potent activity in vitro and in vivo," Cancer Science, vol. 102, no. 7, pp. 1374-1380, 2011.

[13] Z. Jin, X. Cheng, H. Feng et al., "Apatinib inhibits angiogenesis via suppressing akt/gsk3 $\beta / A N G$ signaling pathway in anaplastic thyroid cancer," Cellular Physiology and Biochemistry, vol. 44, no. 4, pp. 1471-1484, 2017.

[14] J. Li, S. Qin, J. Xu et al., "Randomized, double-blind, placebocontrolled phase III trial of apatinib in patients with chemotherapy-refractory advanced or metastatic adenocarcinoma of the stomach or gastroesophageal junction," Journal of Clinical Oncology, vol. 34, no. 13, pp. 1448-1454, 2016.

[15] G. Roviello, A. Ravelli, K. Polom et al., "Apatinib: a novel receptor tyrosine kinase inhibitor for the treatment of gastric cancer," Cancer Letters, vol. 372, no. 2, pp. 187-191, 2016.

[16] Z. Yang, G. Chen, Y. Cui et al., "The safety and efficacy of TACE combined with apatinib on patients with advanced hepatocellular carcinoma: a retrospective study," Cancer Biology \& Therapy, vol. 20, no. 3, pp. 321-327, 2019.

[17] Z. Qiu, L. Shen, S. Chen et al., "Efficacy of apatinib in transcatheter arterial chemoembolization (TACE) refractory intermediate and advanced-stage hepatocellular carcinoma: A propensity score matching analysis: A propensity score matching analysis," Cancer Management and Research, vol. 11, pp. 9321-9330, 2019.

[18] W. Fan, G. Yuan, H. Fan et al., "Apatinib combined with transarterial chemoembolization in patients with hepatocellular carcinoma and portal vein tumor thrombus: a multicenter retrospective study," Clinical Therapeutics, vol. 41, no. 8, pp. 1463-1476, 2019.

[19] G. H. Kim, S. L. Choi, J. H. Kim, J. H. Shim, M. Alali, and N. Kim, "Sorafenib combined with chemoembolization for locally advanced hepatocellular carcinoma with macroscopic vascular invasion: a propensity score analysis," Life (Basel, Switzerland), vol. 11, no. 10, 2021.

[20] C. Koch, M. Göller, E. Schott et al., "Combination of sorafenib and transarterial chemoembolization in selected patients with advanced-stage hepatocellular carcinoma: a retrospective cohort study at three German liver centers," Cancers, vol. 13, no. 9, 2021. 
[21] R. Lencioni and J. M. Llovet, "Modified RECIST (mRECIST) assessment for hepatocellular carcinoma," Seminars in Liver Disease, vol. 30, no. 1, pp. 52-60, 2010.

[22] A.-L. Cheng, Y.-K. Kang, Z. Chen et al., "Efficacy and safety of sorafenib in patients in the Asia-Pacific region with advanced hepatocellular carcinoma: a phase III randomised, doubleblind, placebo-controlled trial," The Lancet Oncology, vol. 10, no. 1, pp. 25-34, 2009.

[23] M. A. Wörns and P. R. Galle, "HCC therapies--lessons learned," Nature Reviews. Gastroenterology \& Hepatology, vol. 11, no. 7, pp. 447-452, 2014.

[24] C. Berasain, "Hepatocellular carcinoma and sorafenib: too many resistance mechanisms?" Gut, vol. 62 , no. 12 , pp. 1674-1675, 2013.

[25] K.-C. Liu, Y.-H. Hao, W.-F. Lv et al., "Transarterial chemoembolization combined with sorafenib in patients with BCLC stage C hepatocellular carcinoma," Drug Design, Development and Therapy, vol. 14, pp. 3461-3468, 2020.

[26] H. H. Chu, J. H. Kim, J. H. Shim, S. M. Yoon, P. H. Kim, and I. Alrashidi, "Chemoembolization plus radiotherapy versus chemoembolization plus sorafenib for the treatment of hepatocellular carcinoma invading the portal vein: a propensity score matching analysis," Cancers, vol. 12, no. 5, 2020.

[27] S. W. Lee, T. Y. Lee, Y. C. Peng, S. S. Yang, H. Z. Yeh, and C. S. Chang, "The therapeutic benefits of combined sorafenib and transarterial chemoembolization for advanced hepatocellular carcinoma," Journal of digestive diseases, vol. 21, no. 5, pp. 287-292, 2020.

[28] T. Pan, X.-S. Li, Q.-K. Xie et al., "Safety and efficacy of transarterial chemoembolization plus sorafenib for hepatocellular carcinoma with portal venous tumour thrombus," Clinical Radiology, vol. 69, no. 12, pp. e553-e561, 2014.

[29] X. Kan, B. Liang, G. Zhou et al., "Transarterial chemoembolization combined with apatinib for advanced hepatocellular carcinoma: a propensity score matching analysis," Frontiers in oncology, vol. 10, p. 970, 2020.

[30] J. Liu, J. Xu, W. Zhang et al., "Safety and efficacy of drugeluting bead transarterial chemoembolization combined with apatinib in patients with advanced hepatocellular carcinoma," Academic Radiology, vol. 27, no. 5, pp. 704-709, 2020.

[31] A. Giobbie-Hurder, R. D. Gelber, and M. M. Regan, "Challenges of guarantee-time bias," Journal of Clinical Oncology, vol. 31, no. 23, pp. 2963-2969, 2013.

[32] J. R. Anderson, K. C. Cain, and R. D. Gelber, "Analysis of survival by tumor response," Journal of Clinical Oncology, vol. 1, no. 11, pp. 710-719, 1983. 